\title{
Influence of conventional antiepileptic drugs on glutathione-S-transferase and lipid peroxidation in children with idiopathic epilepsy
}

\author{
Hamed A. Elkhayat ${ }^{1}$, Hanan M. Hamed ${ }^{2 *}$, Mohamed G. Shouman², Iman A. Elagouza \\ and Lamis H. Mekkawy ${ }^{2}$
}

\begin{abstract}
Background: Evidence suggests that conventional antiepileptic drugs (AEDs) change the oxidative-antioxidative balance in epileptic children, so our aim of this study to evaluate the influence of the conventional AEDs valproic acid (VPA), carbamazepine (CBZ), or both on antioxidant enzyme activity and lipid peroxidation in epileptic children.

Methods: Fifty patients with idiopathic epilepsy and twenty five healthy children serving as controls were included in the study. The epileptic children were further subdivided into three subgroups according to drug therapy: group A, twenty patients treated with VPA; group B, twenty patients treated with $C B Z$; and group $C$, ten patients treated with both drugs. Malondialdehyde (MDA) and glutathione-S-transferase (GST) levels were measured in the patients and controls.

Results: The GST level was significantly lower in the patients than that in the controls $(p<0.001)$, while the MDA level was significantly higher in the patients than that in the controls $(p<0.001)$. The duration of drug intake was negatively correlated with the GST level and positively correlated with the MDA level in the three epileptic patient groups.
\end{abstract}

Conclusion: The oxidant-antioxidant balance is disturbed in epileptic children who receive VPA, CBZ, or both.

Keywords: Epilepsy, Malondialdehyde, Glutathione-S-transferase, Conventional antiepileptic drugs

\section{Background}

Epilepsy is one of the most common neurological disorders; the WHO's 2010 Global Burden of Disease study ranks epilepsy as the second most burdensome neurologic disorder worldwide in terms of disability-adjusted life years (Murray et al. 2012). The prevalence of active epilepsy was estimated to be 6.38 per 1000 persons (95\% CI 5.57-7.30) (Fiest et al. 2017). The prevalence of epilepsy did not differ by age group, sex, or study quality, and incidence rate of epilepsy was higher in low- to middle-income countries (Fiest et al. 2014). Epilepsy is characterized by recurrent unprovoked seizures (Melcon et al. 2007). Epilepsy is usually controlled with conventional antiepileptic drugs (AEDs) but cannot be cured; however, more than $30 \%$ of people with epilepsy do

\footnotetext{
* Correspondence: hmhamed63@yahoo.com

${ }^{2}$ Pediatric Department, National Research Centre, Cairo, Egypt

Full list of author information is available at the end of the article
}

not achieve seizure control, even with the best available medications (George and Krucik 2014).

Evidence suggests that conventional AEDs change the oxidative-antioxidative balance in epileptic children (Cárdenas-R et al. 2013; Aycicek and Iscan 2007).

Conventional AEDs greatly increase lipid peroxidation at the expense of protective antioxidants, leading to an increase in seizure recurrence and idiosyncratic drug effects (Tan et al. 2009). Peroxidation of lipid membranes caused by increased generation of free radicals or decreased activity of antioxidant defense systems has been suggested to critically affect seizure control (Shin et al. 2011). Malondialdehyde (MDA), an end product of lipid peroxidation, is an indicator of free radical metabolism and oxidative stress in humans and other organisms and is altered during administration of AEDs (Ebru et al. 2011). Glutathione is an important biomolecule that protects the cell against chemical-induced cytotoxicity 
by direct or enzymatic glutathione-S-transferase (GST) conjugation with electrophilic compounds and reactive oxygen species (ROS), such as lipid hydroperoxides and hydrogen peroxide, and is affected by anticonvulsant treatments (Cengiz et al. 2000).

Therefore, we aimed to evaluate the influence of conventional AEDs (valproic acid (VPA), carbamazepine (CBZ), or both) on antioxidant enzyme activity and lipid peroxidation in children with idiopathic epilepsy by measuring GST activity and the MDA concentration in both epileptic and healthy children.

\section{Methods}

The present study was a case control study conducted on 50 children and adolescents with idiopathic epilepsy recruited from the Paediatric Neurology Clinic, Children's Hospital, Ain Shams University. Their ages ranged from 2 to 14 years, and the patients included 27 males and $23 \mathrm{fe}$ males. The patients were classified into three groups according to the type of AED received:

Group A: It included 20 epileptic patients (14 males and 6 females) treated with VPA monotherapy.

Group B: It included 20 epileptic patients (8 males and 12 females) treated with CBZ monotherapy.

Group C: It included 10 epileptic patients $(5$ males and 5 females) treated with both VPA and CBZ.

Exclusion criteria were any patients with progressive neurological disease, with confirmed neurodegenerative disorders, or with a disease affecting the serum levels of GST and MDA, such as chronic liver disease. Twenty-five healthy children age- and sex-matched were recruited as control group.

A full history was taken for each patient with an emphasis on epilepsy type and age of onset of seizures and frequency. Seizure control was considered good if the seizure frequency was $\leq 2$ times/month. However, a seizure frequency $>4$ times/month was considered uncontrolled. The severity of seizures was rated according to the Chalfont seizure severity scale (Duncan and Sander 1991). AEDs (number, type, duration and dosage of each drug, and its effect on seizure control) were recorded. A thorough clinical examination was performed for all patients.

In all studied groups, $5 \mathrm{ml}$ of venous blood was collected under aseptic conditions. The blood sample was divided into two parts:

(1) The first part was allowed to clot at room temperature and was then centrifuged at $1000 \mathrm{rpm}$ for $2 \mathrm{~min}$. The serum was then collected to determine the liver function, kidney function, and serum GST level.

(2) The second part was collected in a tube containing EDTA; $0.5 \mathrm{ml}$ was used for a complete blood picture $(\mathrm{CBC})$, and the remaining sample was centrifuged. The separated plasma was then used to determine the plasma MDA level.

The $\mathrm{CBC}$ and liver and kidney functions were measured to exclude any associated illness or drug side effects. Electroencephalography (EEG), computed tomography (CT), and/ or magnetic resonance imaging (MRI) were performed to reveal subtle structural abnormalities and identify lesions.

GST activity was measured using 1-chloro-2,4-dinitrobenzene $(\mathrm{CDNB})$, which is a synthetic GST substrate. Briefly, CDNB was added to a buffer containing GSH and an aliquot of sample to be tested. Upon the addition of CDNB, the change in absorbance at $340 \mathrm{~nm}$ was measured as a function of time. The results are expressed in units per liter (Habig and Jakoby 1981).

MDA level was determined by the reaction of MDA with thiobarbituric acid (TBA) at $95{ }^{\circ} \mathrm{C}$. In the TBA test reaction, MDA and TBA react to form a pink pigment with an absorption maximum at $532 \mathrm{~nm}$. The reaction was performed at $\mathrm{pH} 2-3$ at $95{ }^{\circ} \mathrm{C}$ for $15 \mathrm{~min}$. The sample was mixed with 2.5 volumes of $10 \%(w / v)$ trichloroacetic acid to precipitate the protein. The precipitate was pelleted by centrifugation, and an aliquot of the supernatant was allowed to react with an equal volume of $0.67 \%$ TBA in a boiling water bath for $15 \mathrm{~min}$. After cooling, the absorbance was read at $532 \mathrm{~nm}$. The arbitrary values obtained were compared with a series of standard solutions (1,1,3,3-tetramethoxypropane). The results are expressed as nanomoles per milliliter (Draper and Hadley 1990). None of the patients had received a blood transfusion within 2 weeks before blood sampling, as this may interfere with the serum levels of GST and MDA.

\section{Statistical methods}

The statistical package SPSS version 15 for Windows (SPSS, Chicago, IL, USA) was used for the statistical analysis. Data are represented as the means \pm standard deviations or frequencies and percentages. Student's $t$ test was used to compare two independent means. For comparing categorical data, chi-square $\left(\chi^{2}\right)$ test was performed. One-way ANOVA was used to compare three or more independent means, followed by a post hoc test when a significant difference was found. Correlation was estimated by Pearson's correlation coefficient. A $p$ value $<0.05$ was considered statistically significant.

\section{Results}

The study included 50 patients (27 males and 23 females) with a mean age of $5.6 \pm 3.3$ years as well as 25 healthy controls (12 males and 13 females) with a mean age of 6.9 \pm 2.9 years. There were no significant differences in sex distribution among the patient subgroup and controls $\left(x^{2}=1.7\right.$ and $\left.p=0.3\right)$. 
The demographic and laboratory data of the patients and controls are presented in Table 1. The GST level was significantly lower in the patients than in the controls $(p<0.001)$, while the MDA level was significantly higher in the patients than in the controls $(p<0.001)$ (Table 1). No significant difference was noted in GST and MDA levels among the patient subgroups (Fig. 1).

Based on the number of seizures per month, we classified the patients into controlled and poorly controlled groups ( $>4$ times/month). A significantly higher serum GST level was observed in the patients with controlled seizures than that in the patients with poor seizure control, whereas a significantly higher serum MDA level was found in the patients with poor seizure control than in the patients with controlled seizures (Table 2). No statistically significant differences were found in the seizure frequency and Chalfont severity score, while the duration of drug therapy was negatively correlated with the GST level and positively correlated with the MDA level in the three groups of epileptic patients (Table 3).

\section{Discussion}

Evidence suggests that glutathione homeostasis may be altered due to reactive metabolites and/or ROS produced during treatment with anticonvulsants (Cárdenas- $\mathrm{R}$ et al. 2013). Glutathione is an important biomolecule that protects the cell against chemical-induced cytotoxicity by direct or enzymatic (GST) conjugation with electrophilic

Table 1 The demographic and laboratory data of the studied children

\begin{tabular}{lllc}
\hline & Patients & Controls & $p$ value \\
\hline Age (years) & $5.6 \pm 3.3$ & $6.9 \pm 2.9$ & 0.9 \\
Sex (males/females) & $27 / 23$ & $12 / 13$ & 0.6 \\
BMI (kg/m ${ }^{2}$ ) & $20.7 \pm 4.16$ & $19.5 \pm 3.5$ & 0.2 \\
Age of onset (years) & $5.5 \pm 3.6$ & & \\
Duration of the & $2.87 \pm 1.87$ & & \\
therapy (years) & & & \\
Frequency of & $2.22 \pm 1.037$ & & \\
seizure/month & & & \\
Type of seizure: & & & \\
Focal & & & \\
$\quad$ Simple partial & $5(10 \%)$ & & \\
Complex partial & $14(28 \%)$ & & \\
Generalized & & & \\
Tonic clonic & $29(58 \%)$ & & \\
Myoclonic & $2(4 \%)$ & & \\
Uncontrolled/ & $18 / 32$ & & \\
controlled seizure & & & \\
Chalfont severity scale & $28.38 \pm 26.06$ & & \\
MDA (nmol/ml) & $2.3 \pm 0.3$ & & \\
GST (U/l) & $835.2 \pm 200.6$ & $1537.6 \pm 298.7$ & \\
\hline
\end{tabular}

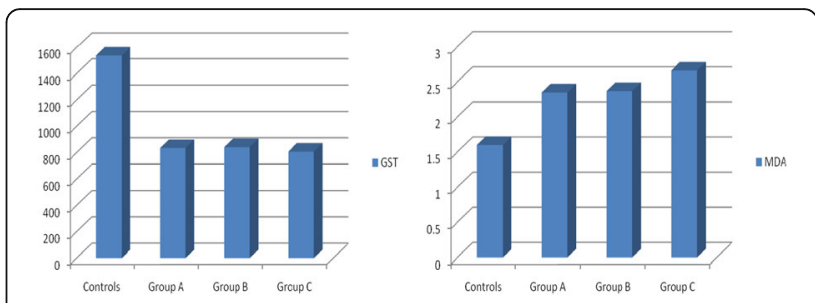

Fig. 1 Comparison of the GST and MDA levels among the different patient groups and control. Group A: patients on valproate monotherapy; Group B: patients on carbamazepine monotherapy; Group C: patients on valproate and carbamazepine. MDA Malondialdehyde, GST glutathione-S-transferase

compounds while demonstrating reactive oxygen teratogenic potential in patients with epilepsy (Ebru et al. 2011).

In the current study, the GST level was significantly decreased in the epileptic patients compared to that in the control group; however, no significant difference was found among the different patient subgroups. These results are consistent with the findings of studies by Michoulas et al. (2006) and Solowiej and Sobaniec (2003). Solowiej and Sobaniec (2003) reported that the intermediate metabolites of VPA and CBZ are detoxified via conjugation with glutathione, leading to the efflux of GST in bile. Thus, depletion of hepatic GST stores as a result of this detoxification might account for the changes observed in the serum GST level. However, Adnan et al. (2001) recorded a decrease in the GST level in patients treated with VPA, while it was not changed in patients receiving CBZ. They stated that CBZ may be a better drug for management of epilepsy, as its metabolic intermediates were more stable and thus resulted in less free radical injury than VPA.

Children with idiopathic epilepsy treated with VPA and/ or CBZ have a disrupted oxidant-antioxidant balance and thus require an adequate supply of antioxidants for brain protection and prevention of neurological disturbances. In addition, long-term use of valproate could lead to a lack of selenium, copper, and zinc, which are cofactors of GST activity. Thus, long-term administration of AEDs in epileptic children leads to decreased GST levels (Adnan et al. 2001).

Graf et al. (1998) suggested that deficits in antioxidant activity enhance susceptibility to the adverse effects of VPA therapy. Moreover, another study reported no

Table 2 Comparison of the GST and MDA levels among the patients with controlled seizures and those with uncontrolled seizures

\begin{tabular}{lllll}
\hline Items & Group & Number & Means \pm SD & $p$ value \\
\hline GST (U/l) & Uncontrolled & 18 & $759.01 \pm 162$ & $<0.001$ \\
& Controlled & 32 & $918.1 \pm 198$ & \\
MDA (nmol/mL) & Uncontrolled & 18 & $2.54 \pm 0.25$ & $<0.001$ \\
& Controlled & 32 & $2.19 \pm 0.23$ & \\
\hline
\end{tabular}


Table 3 Correlation between the duration of drug therapy and level of glutathione-S-transferase and malondialdehyde in the epileptic groups

\begin{tabular}{llll}
\hline Group & & $\begin{array}{l}\text { MDA } \\
(\mathrm{nmol} / \mathrm{mL})\end{array}$ & $\begin{array}{l}\text { GST } \\
(\mathrm{U} / \mathrm{l})\end{array}$ \\
\hline Group A & $r$ & 0.663 & -0.730 \\
& $p$ value & 0.001 & 0.001 \\
Group B & $r$ & 0.621 & -0.749 \\
& $p$ value & 0.003 & 0.001 \\
Group C & $r$ & 0.840 & -0.917 \\
& $p$ value & 0.002 & 0.001
\end{tabular}

Group A: patients on valproate monotherapy

Group B: patients on carbamazepine monotherapy

Group C: patients on valproate and carbamazepine

MDA malondialdehyde, GST glutathione-S-transferase

change in the glutathione peroxidase level in erythrocytes during treatment with VPA (Yiş et al. 2009).

In contrast to our results, Kürekçi et al. (1995) found that serum GST levels were increased in patients on VPA therapy. They reported that this increase might be due to an induction of hepatic synthesis of glutathione.

Lipid peroxidation is an indicator of free radical metabolism and oxidative stress in humans and other organisms. Free radicals have a short half-life, and the estimation of MDA, which is an end product of lipid peroxidation formed during the non-enzymatic oxidation of polyunsaturated fatty acids, is both simple and convenient for determining lipid peroxidation and thereby oxidative stress in human subjects (Waldbaum and Patel 2010).

Excess oxidative stress may be a final common pathway through which AEDs exert their teratogenic potential in epilepsy patients (Ebru et al. 2011).

Our results showed that the MDA level was significantly higher in the patients with idiopathic epilepsy than in the controls. This finding is consistent with that of Michoulas et al. (2006). No significant difference in the MDA level was detected among our patient subgroups. Zhang et al. (2011) and Bindu et al. (2012) reported an increased MDA level in epileptic patients receiving VPA compared to that in the control group. Solowiej and Sobaniec (2003) reported a decreased MDA level in patients treated with CBZ monotherapy, while the MDA level was elevated in patients treated with VPA. This might be due to the decreased activities of the antioxidant enzymes associated with VPA therapy, whereas these enzymes showed normal activity during CBZ therapy (Ebru et al. 2011). Yiş et al. (2009) reported that the MDA levels were significantly increased but did not reach pathological levels in epileptic patients receiving VPA.

In contrast to our results, Yüksel et al. (2000) detected no significant difference in serum MDA levels between epileptic children treated with VPA and healthy controls.
Changes in the antioxidant defense mechanisms and the resulting lipid peroxidation are involved in the pathogenesis of epilepsy. However, research findings concerning these processes are very conflicting (Solowiej and Sobaniec 2003).

In the present study, the serum GST level was significantly lower in the poor seizure control group (for at least 6 months) than in the controlled seizure group. However, the serum MDA level was significantly higher in the patients with poor seizure control than in the patients who were controlled. Our results were consistent with those of Graf et al. (1998) who reported a normal GST level in children showing good clinical tolerance and a decreased level in patients with repeated seizures. Their study showed that antioxidant enzymes including GST were saturated when the free radical load of the body increased. They also demonstrated that when these enzymes failed to compensate for the excessive repeated free radical load due to repeated seizures, the antioxidant enzyme level started to decrease, while damage occurred within the cell membranes. They added that VPA especially increased the oxygen-associated tissue damage due to its toxic metabolites in the body.

The duration of drug intake was negatively correlated with the GST level and positively correlated with the MDA level in the three groups of epileptic patients. Zhang et al. (2011) reported a positive correlation between the plasma MDA level and the duration of VPA treatment. Additionally, Martinc et al. (2012) found that erythrocyte GST activity was markedly decreased after long-term administration of VPA. Liu et al. (1998) correlated long-term use of various AEDs to deficiencies in magnesium and serum zinc, which play a critical role in neuronal excitability, in red blood cells.

A limitation of this study is the small size of the patients.

\section{Conclusions}

The oxidant-antioxidant balance is disturbed in epileptic patients treated with VPA, CBZ, or both. These imbalances are affected by both the duration of the drug treatment and epilepsy control.

\section{Recommendations}

We recommend a large-scale study measuring both GST and MDA in newly diagnosed idiopathic epilepsy patients before and after treatment with VPA and/or CBZ. The effect of antioxidants on GST and MDA levels opens a new therapeutic window for epilepsy patients.

Acknowledgements

We acknowledge the National Research Centre that funded this work. 


\section{Funding}

This work was funded by the National Research Centre.

\section{Availability of data and materials}

All the data of patients are available.

\section{Authors' contributions}

This work was carried out in collaboration between all authors. HAE, MGS, and IAAE designed the study and wrote the protocol. HMH managed the literature searches. LH and IAAE managed patients' recruitment. $\mathrm{HMH}$ performed the statistical analysis. All the authors wrote, revised, edited, and managed the analyses of the study and executed the manuscript in its final form. All authors read and approved the final manuscript.

\section{Ethics approval and consent to participate}

Informed consent was obtained from the parents and/or caregivers of all participants before the procedure, according to the guidelines of the ethical committee.

\section{Consent for publication}

I am and all authors have approved the manuscript for submission to your journal hoping that it is going to be accepted for publication.

\section{Competing interests}

The authors declare that they have no competing interests.

\section{Publisher's Note}

Springer Nature remains neutral with regard to jurisdictional claims in published maps and institutional affiliations.

\section{Author details}

${ }^{1}$ Pediatric Department, Faculty of Medicine, Ain Shams University, Cairo, Egypt. ${ }^{2}$ Pediatric Department, National Research Centre, Cairo, Egypt.

Received: 18 June 2018 Accepted: 25 September 2018

Published online: 03 November 2018

\section{References}

Adnan Y, Mujgan C, Mehmet T, Turjut U (2001) Changes in the antioxidant system in epileptic patients receiving AEDs. J Child Neuro 16:603-605

Aycicek A, Iscan A (2007) The effects of carbamazepine, valproic acid and phenobarbital on the oxidative and antioxidative balance in epileptic children. Eur Neurol 57:65-69

Bindu M, Krishnan R, Rajendiran V (2012) Oxidative stress in patients with epilepsy is independent of antiepileptic drugs. Seizure 21(10):780-784

Cengiz M, Yuksel A, Seven M (2000) The effects of carbamazepine and valproic acid on the erythrocyte glutathione, glutathione peroxidase, superoxide dismutase and serum peroxidation in epileptic children. Pharmacol Res 41:423-425

Draper HH, Hadley M (1990) Malondialdehyde determination as index of lipid peroxidation. Methods Enzymol 186:421-431

Duncan JS, Sander JW (1991) The Chalfont Seizure Severity Scale. J Neurol Neurosurg Psychiatry 54(10):873-876

Ebru A, Ayse S, Bahadır O, Ozturk HS, Aysima O, Nese K, Ebru K, Neriman S (2011) Effects of epilepsy and antiepileptic drugs on nitric oxide, lipid peroxidation and xanthine oxidase system in children with idiopathic epilepsy. Seizure 20:138-142

Fiest KM, Birbeck GL, Jacoby A, Jette N (2014) Stigma in epilepsy. Curr Neurol Neurosci Rep 444:14 [PubMed]

Fiest KM, Sauro KM, Wiebe S, Patten SB, Kwon CS, Dykeman J, Pringsheim T, Lorenzetti DL, Jetté N (2017) Prevalence and incidence of epilepsy: a systematic review and meta-analysis of international studies. Neurology 88(3): 296-303. https://doi.org/10.1212/WNL.0000000000003509

George T, Krucik (2014) Long-term prognosis for epilepsy. Epilepsy 201;55(8): 1213-1219. https://doi.org/10.1111/epi.12699

Graf W D, Oleinik O E, Glauser TA, Maertens P, Eder D N, Pippenger C .Altered antioxidant activities in children with a serious side adverse experience related to valproic acid therapy. Neuropediatrics;1998 29: 195-201

Habig WH, Jakoby WH (1981) Assays for differentiation of glutathione Stransferase. Methods Enzymol 77:398-405
Kürekçi AE, Alpay F, Tanindi S, Gokcay E, Ozcan O, Akin R (1995) Plasma trace element, plasma glutathione peroxidase, andsuperoxide dismutase levels in epileptic children receiving AEDs therapy. Epilepsia 36:600-604

Liu CS, Wu HM, Kao SH, Wei YH (1998) Serum trace elements, glutathione, copper/zinc superoxide dismutase, and lipid peroxidation in epileptic patients with phenytoin or carbamazepine monotherapy. Clin Neuropharmacol 21(1):62-64 [PubMed]

Martinc B, Grabnar I, Vovk T (2012) The role of reactive species in epileptogenesis and influence of antiepileptic drug therapy on oxidative stress. Curr. Neuropharmacol 10(4):328-343. https://doi.org/10.2174/157015912804499447 [PMC free article] [PubMed] [Cross Ref]

Melcon MO, Kochen S, Vergara RH (2007) Prevalence and clinical features of epilepsy in Argentina. A community-based study. Neuroepidemiology 28(1):8-15

Michoulas A, Tong V, Teng XW, Chang TK, Abbott FS, Farrell K (2006) Oxidative stress in children receiving valproic acid. J. Pediatr 149(5):692-696. https://doi.org/10. 1016/j.jpeds.2006.08.015 [PubMed][Cross Ref]

Murray CJ, Vos T, Lozano R (2012) Disability-adjusted life years (DALYs) for 291 diseases and injuries in 21 regions, 1990-2010: a systematic analysis for the Global Burden of Disease Study 2010. Lancet 380:2197-2223 [PubMed]

Noemí Cárdenas-R, Elvia C U, Liliana RE, Arantxa RT, Aristides S, Daniel O C. Modulation of antioxidant enzymatic activities by certain antiepileptic drugs (Valproic acid, Oxcarbazepine, and Topiramate): evidence in humans and experimental models. Oxidative Medicine and Cell Longevity2013.Article ID 598493. https://doi.org/10.1155/2013/598493

Shin E, Jeong HJ, Chung YH (2011) Role of oxidative stress in epileptic seizures. Neurochem Int 59:122-137

Solowiej E, Sobaniec W (2003) The effect of antiepileptic drug therapy on antioxidant enzyme activity and serum lipid peroxidation in young patients with epilepsy. Neurol Neuroch Pol 37:991-1003

Tan TY, Lu CH, Chuang HY, Lin TK, Liou CW, Chang WN et al (2009) Long-term antiepileptic drug therapy contributes to the acceleration of atherosclerosis. Epilepsia 50:1579-1586

Waldbaum S, Patel M (2010) Mitochondrial dysfunction and oxidative stress: a contributing link to acquired epilepsy? J. Bioenerg. Biomembr 42(6):449-455. https://doi.org/10.1007/s10863-010-9320-9 [PMC free article] [PubMed] [Cross Ref]

Yiş U, Seçkin E, Kurul SH, Kuralay F, Dirik E (2009) Effects of epilepsy and valproic acid on oxidant status in children with idiopathic epilepsy. Epilepsy Res 84(2-3):232-237

Yüksel A, Cengiz M, Seven M, Ulutin T et al (2000) Erythrocyte glutathione, glutathione peroxidase, superoxide dismutase and serum lipid peroxidation in epileptic children with valproate and carbamazepine monotherapy. J Basic Clin Physiol Pharmacol 11(1):73-81. PubMed

Zhang YJ, Zhang M, Wang XC, Yu YH, Jin PJ, Wang Y (2011) Effects of sodium valproate on neutrophils' oxidative metabolism and oxidant status in children with idiopathic epilepsy. Zhonghua Er Ke Za Zhi 49(10):776-781

\section{Submit your manuscript to a SpringerOpen ${ }^{\circ}$ journal and benefit from:}

- Convenient online submission

- Rigorous peer review

- Open access: articles freely available online

- High visibility within the field

- Retaining the copyright to your article

Submit your next manuscript at $>$ springeropen.com 